\title{
Studies on Physicochemical Characteristics of Anthocyanin from Super Dark Maize
}

\author{
Xiupeng Mei, Hongni Qin, Jiuguang Wang, Guoqiang Wang, Chaoxian Liu, Yilin Cai* \\ Maize Research Institute, Key Laboratory of Biotechnology and Crop Quality Improvement, Ministry of Agriculture, Southwest \\ University, Chongqing, China \\ *Corresponding author: caiyilin1789@163.com
}

Received December 26, 2013; Revised March 25, 2014; Accepted March 30, 2014

\begin{abstract}
The effects of temperature, light, oxidant, soluble sugar, food additive (i.e. citric acid, benzoic acid, and ascorbic acid) and metal ions of $\mathrm{K}^{+}, \mathrm{Ca}^{2+}, \mathrm{Cu}^{2+}, \mathrm{Al}^{3+}, \mathrm{Mn}^{2+}, \mathrm{Fe}^{3+}, \mathrm{Sn}^{2+}, \mathrm{Mg}^{2+}$ on the stability of anthocyanin extracted from the kernels of novel inbred line super dark maize (SDM) with the strategy of $\mathrm{HCl}$-carbinol were systematically analyzed. The results showed that the extracted anthocyanin was steady in acid conditions $(\mathrm{pH} \leq 3)$ and stable to heat and light; it had good anti-oxidation and maintained sensitive to reducing agents and insensitive to soluble sugar. Citric acid had positive effect on the stability of anthocyanin while benzoic acid and ascorbic acid negative; metal ions $\left(1 \mathrm{mMol} \cdot \mathrm{L}^{-1}\right)$ of $\mathrm{K}^{+} 、 \mathrm{Ca}^{2+} 、 \mathrm{Cu}^{2+} 、 \mathrm{Al}^{3+} 、 \mathrm{Mn}^{2+}$ exhibited no obvious effect on the anthocyanin while ions of $\mathrm{Fe}^{3+} 、 \mathrm{Sn}^{2+} 、 \mathrm{Mg}^{2+}$ obvious.
\end{abstract}

Keywords: anthocyanin, physicochemical characteristics, super dark maize

Cite This Article: Xiupeng Mei, Hongni Qin, Jiuguang Wang, Guoqiang Wang, Chaoxian Liu, and Yilin Cai, "Studies on Physicochemical Characteristics of Anthocyanin from Super Dark Maize." Journal of Food and Nutrition Research, vol. 2, no. 3 (2014): 109-114. doi: 10.12691/jfnr-2-3-3.

\section{Introduction}

Anthocyanin, belonging to flavonoids as plant secondary metabolite, exists ubiquitously in the plant kingdom and endows fruits and vegetables with the bright red, blue and purple color $[1,2,3]$. It was recognized as a functional compound for human health and was utilized extensively in food and medicine industries. Particularly, it exhibited positive functions in inhibiting free radicals, stimulation of phase II detoxifying enzymes, and reducing the risk of cardiac diseases and cancers by restraining cell proliferation [4,5,6,7]. Furthermore, another research showed that regular consumption of flavonoids may increase human life-span by reducing inflammation and coronary heart disease (CHD) [8,9,10].

Numerous researches had been done to black maize which is a kind of excellent natural pigment resources. As the main pigment composition of the black maize kernels, anthocyanin possesses complicated physical and chemical properties due to its transformable structure. Some properties were described in different materials. The antioxidant activity and the effect of light and temperature had been demonstrated on the anthocyanin of kernel and cob in maize [11-16]; and the research on the effect of preservative, redox and metal ions on anthocyanin had also been mentioned [17]. Previous studies had confirmed that anthocyanin played an important role in maintaining human health. Therefore it is necessary to develop new resources rich in anthocyanin for the daily supply.
A completely new black maize inbred line named Super Dark Maize (SDM) was created by Maize Research Institute of Southwest University (Chongqing, China) in recent years. Its kernels and cobs were a utter inkiness and were notably distinguished from normal black maize with mere black top of kernels. The anthocyanin content of the SDM kernels was 12 times as much as that of the normal black maize. The property analysis of anthocyanin was prerequisite for the SDM kernels exploitation and utilization. Therefore, a systematic analysis was done to the physicochemical characteristics of anthocyanin in SDM kernels. The effects of $\mathrm{pH}$, temperature, light, oxidant, soluble sugar, food additives and metal ions on anthocyanin stability were investigated in this paper. The absorbance or residual rate of anthocyanin in different time was made as a criterion based on the $\mathrm{pH}$ differential method.

\section{Materials and Methods}

\subsection{Sample Preparation}

For the test sample preparation, dry maize kernels were soaked in distilled water for 24 hours, the purple or dark episperm were collected. After the episperm was dried in oven at constant temperature $55^{\circ} \mathrm{C}, 0.1 \mathrm{~g}$ dried sample was put in the centrifuge tube, then $5 \mathrm{~mL} \mathrm{5 \%}$ hydrochloric acid-methanol solution was added to the tube. The tube was water bathed for 1 hour at $50^{\circ} \mathrm{C}$ and then centrifuged for 5 minutes at $10000 \mathrm{r} / \mathrm{min}$. The extracted supernatant was metered to a volume of $15 \mathrm{~mL}$. The anthocyanin 
solution was scaned in the wavelength 200-800 nm using the UV1000 Spectrophotometer. Then the anthocyanin absorbance was measured in the maximum wavelength (507 nm).

The $\mathrm{pH}$ differential method was adopted to calculate the mass fraction of anthocyanin [18,19,20]. $20 \mathrm{~mL}$ sample solution in total was prepared for analysis and $3 \mathrm{~mL}$ sample solution was used for every colorimetric assay and not recycled. $\mathrm{KCl}(\mathrm{pH} 1.0)$ and $\mathrm{NaAc}(\mathrm{pH}$ 4.5) buffer solutions were used to metered to a volume of $10 \mathrm{~mL}$ independently. Moreover, the control groups were made by using another $10 \mathrm{~mL}$ corresponding absolute buffer solution. Absorbance of the anthocyanin was measured in the wave length $507 \mathrm{~nm}$ and $700 \mathrm{~nm}$ respectively. The mass fraction of anthocyanin was calculated by the formula $M F=\frac{A \times V \times N \times M}{\varepsilon \times m}$, in which MF representes mass fraction of anthocyanin $\left(\mathrm{mg} \cdot \mathrm{g}^{-1}\right), A=\left(A_{\max }-A_{700}\right)_{\mathrm{pH} 1.0^{-}}$ $0.1\left(A_{\max }-A_{700}\right)_{\mathrm{pH} 4.5}, V$ means determined volume $(\mathrm{mL}), N$ means dilute multiple, $M$ and $\varepsilon$ means molecular weight and extinction coefficient of Cyanidin-3-glucoside respectively, m means sample weight $(\mathrm{g})$.

\section{2. pH Stability Analysis}

The sample solutions with $\mathrm{pH}$ value of $1,3,5,7,9,12$ were darkly kept in $4^{\circ} \mathrm{C}$ refrigerator. The absorbance $A$ was measured and the residual rate of anthocyanin was calculated using formula $\mathrm{R}=\left(\mathrm{MF} / \mathrm{MF}_{0}\right) \times 100 \%$ every 5 days, in which MF represented mass fraction of anthocyanin after reaction, $\mathrm{MF}_{0}$ represented mass fraction of anthocyanin initially.

\subsection{Light and Thermal Stability}

For the light stability assay, corresponding solutions were darkly and brightly kept for 30 days at $4^{\circ} \mathrm{C}$ separately, the absorbance $A$ was measured and the residual rate of anthocyanin was calculated every 5 days. For the thermal stability assay, every sample solution was incubated for 2 hours by the water bath in darkness at $30^{\circ} \mathrm{C}, 50^{\circ} \mathrm{C}, 60^{\circ} \mathrm{C}, 80^{\circ} \mathrm{C}, 100^{\circ} \mathrm{C}$ respectively, then the solution absorbance $A$ was determined with colorimetric method after cooling.

\subsection{Antioxidant and Anti-reducing Agent Capacity Assay}

The sample solutions darkly kept at $4^{\circ} \mathrm{C}$ with different concentrations of $\mathrm{H}_{2} \mathrm{O}_{2}(0.03 \%-3 \%)$ and $\mathrm{Na}_{2} \mathrm{SO}_{3}(0.2 \times 10$ $\left.{ }^{4}-3 \times 10^{-3} \mathrm{~mol} / \mathrm{L}\right)$ were designed for antioxidant and antireducing agent capacity assays separately. Subsequently, each solution absorbance and residual rate of anthocyanin were measured and calculated after 0.5, 2, 5, 10 hours respectively.

\subsection{Effects of Soluble Sugar and Food Additives}

The sample solutions with different concentrations $(0.03 \%-3 \%)$ of glucose, maltose, sucrose were used to analyze the effects of soluble sugar on anthocyanin. And for the effects of food additives assays, the sample solutions with different concentrations of ascorbic acid (0.5\%-10\%), citric acid and benzoic acid (0.04\%-3\%) were prepared. All sample solutions were darkly kept at $4^{\circ} \mathrm{C}$ and the absorbance and residual rate of anthocyanin were determined every 5 days.

\subsection{Effects of Metal Ions on Anthocyanin Stability}

The sample solutions with $1 \mathrm{mMol} \cdot \mathrm{L}^{-1}$ metal ions $\mathrm{Fe}^{3+}$, $\mathrm{Sn}^{2+}, \mathrm{K}^{+}, \mathrm{Ca}^{2+}, \mathrm{Cu}^{2+}, \mathrm{Mg}^{2+}, \mathrm{Al}^{3+}, \mathrm{Mn}^{2+}$ and check were darkly stored in $4^{\circ} \mathrm{C}$. The absorbances of different treatments were measured on $0.5,2,5,10$ hours separately.

\subsection{Statistical Analysis}

Statistical analysis and charting for all physical and chemical properties of anthocyanin were conducted by using Microsoft Office Excel 2010.

\section{Result and Discussion}

\section{1. pH Stability}

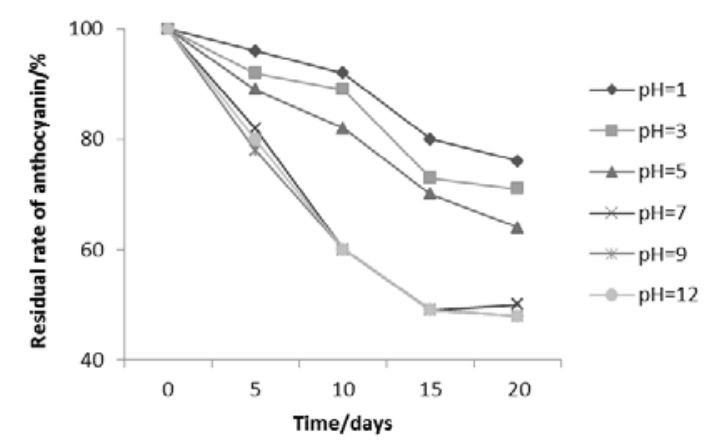

Figure 1. Change of residual rate of anthocyanin with time under different $\mathrm{pH}$ values

To elaborate the $\mathrm{pH}$ stability of anthocyanin in SDM kernels, the $\mathrm{pH}$ profile of anthocyanin stability was determined from $\mathrm{pH} 1.0$ to $\mathrm{pH}$ 12. The residual rate exhibited a gentle decrease when the solution with the $\mathrm{pH}$ value of 1,3 had been placed for 10 days and a similar linear decrease when the solution with $\mathrm{pH}$ value of $5,7,9$, 12 had been placed for 15 days (Figure 1). The notable difference of the residual rate in acidic and alkaline conditions indicated that the anthocyanin was more stable in acidic conditions, especially in $\mathrm{pH} \leq 3$. Additionally, the residual rate of anthocyanin was less than $50 \%$ when solution $\mathrm{pH}>7$, while residual rates were $76 \%, 71 \%$, and $64 \%$ respectively when $\mathrm{pH}$ values were $1,3,5$ on the 20 th day.

The structures of anthocyanin were transformed with the changes of $\mathrm{pH}$ values in the solution medium and maintained an equilibrium state including four main kinds of structures in particular $\mathrm{pH}$ value [14]. The specific characteristics of structures endow anthocyanin with relatively complex and quite unstable physical and chemical properties $[21,22,23,24]$. In the involvement of the multitudinous degradation progresses of anthocyanin, the chalcone route was experienced in most situations; however, the intermediate product chalcone degradated into secondary products rapidly (i.e. benzoic acid and 2,4,6-Trihydroxybenzaldehyde) for its unstable structure 
[19]. In our results, the remarkable decrease of residual rate in alkaline conditions might be led by the changed structures depending on the raising $\mathrm{pH}$ value.

\subsection{Light and Thermal Stability}

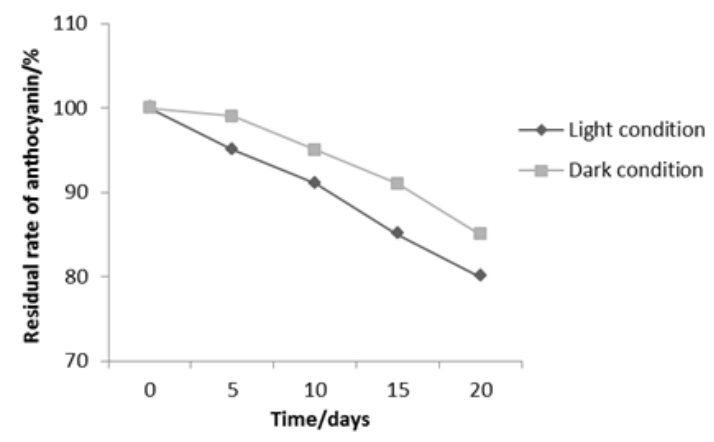

Figure 2. Change of residual rate of anthocyanin with time under different light conditions

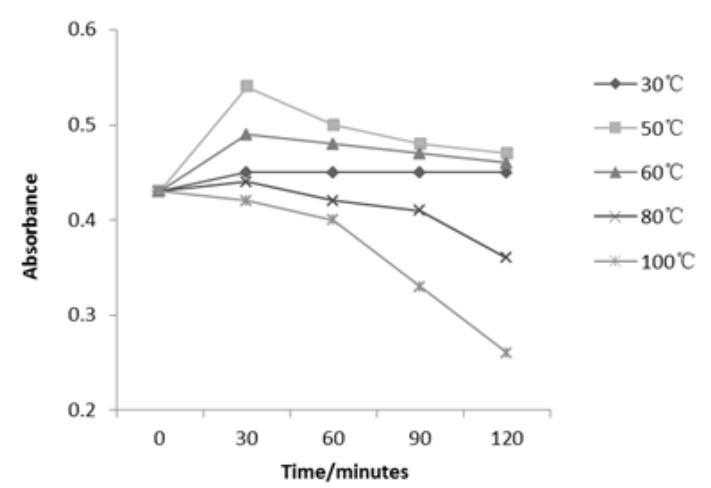

Figure 3. Change of absorbance of anthocyanin with time under different temperatures

The light stability of anthocyanin in SDM kernels was investigated under the lightness and darkness conditions. As shown in Figure 2, in darkness condition, the residual rate of anthocyanin barely changed for the first 5 days. But in lightness condition, the residual rate of anthocyanin decreased to a certain extent on the $5^{\text {th }}$ day. In both conditions, the residual rates of anthocyanin rapidly decreased over 5 days, which indicated that long-term storage for anthocyanin was inappropriate and it would be better to avoid light.

The thermal stability of anthocyanin was assayed between $30^{\circ} \mathrm{C}$ and $100^{\circ} \mathrm{C}$ (Figure 3). The anthocyanin absorbance basically unchanged with the extension of incubation time at $30^{\circ} \mathrm{C}$. At $50^{\circ} \mathrm{C}$ or $60^{\circ} \mathrm{C}$, the absorbance increased in some extent. The maximum value appeared on $30^{\text {th }}$ minute. At $80^{\circ} \mathrm{C}$ or $100^{\circ} \mathrm{C}$, the absorbance of anthocyanin slightly decreased for the first 30 minutes, whereas that of anthocyanin rapidly decreased between 30 to 120 minutes. These results indicated that anthocyanin had a good thermo stability and the formation of anthocyanin was promoted by heating properly.

Light and temperature played an important role in keeping the stability of anthocyanin structure [25]. In light condition, the intermediate product $\mathrm{C}_{4}$ hydroxyl glycosides were prior to be formed. Next, the chalcone and the ultimate products benzoic acid were generated depending on hydrolysis and unlocking loops at the point of $C_{2}$ [19]. For the effect of temperature on anthocyanin, the absorbance was augmented with the increasing temperature at a given range $\left(<60^{\circ} \mathrm{C}\right)$ due to the dissolving rate of anthocyanin was amplified in the solution. In contrast, the absorbance declined because of the extremely unstable chalcone type structures that generated effortlessly at exorbitant temperature.

\subsection{Antioxidant and Anti-reducing Agent Capacity}

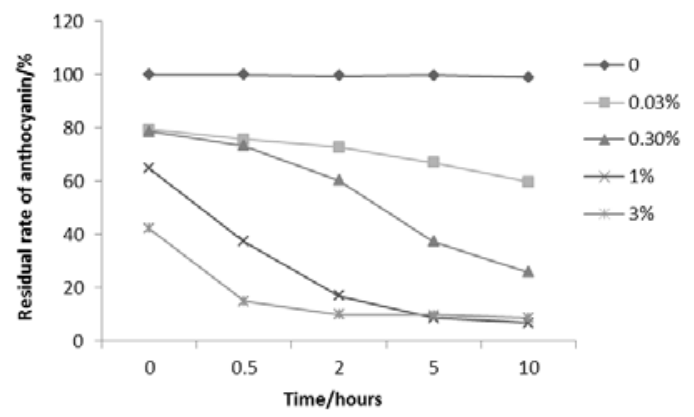

Figure 4. Change of residual rate of anthocyanin with time under different densities of $\mathrm{H}_{2} \mathrm{O}_{2}$

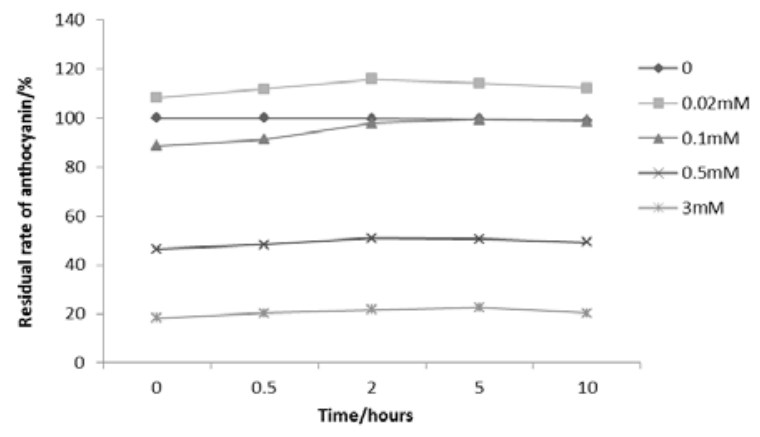

Figure 5. Change of residual rate of anthocyanin with time under different densities of $\mathrm{Na}_{2} \mathrm{SO}_{3}$

The results showed that the $\mathrm{H}_{2} \mathrm{O}_{2}$ and $\mathrm{Na}_{2} \mathrm{SO}_{3}$ influenced anthocyanin solution instantly. The residual rate of anthocyanin continuously decreased with increasing time duration and $\mathrm{H}_{2} \mathrm{O}_{2}$ density, whereas that of anthocyanin changed unobviously along with the time continuation in the analysis of $\mathrm{Na}_{2} \mathrm{SO}_{3}$. The residual rate of anthocyanin differed slightly for $0.03 \%, 0.3 \%$ of $\mathrm{H}_{2} \mathrm{O}_{2}$ in first 0.5 hour, but approximate $15 \%$ anthocyanin was left for $3 \%$ of $\mathrm{H}_{2} \mathrm{O}_{2}$ at 0.5 hour (Figure 4). These results indicated that the decomposition of anthocyanin could be accelerated by adding oxidant and the degradation rate was proportional to the concentration of oxidant, which was consistent with Neungnapa's result [13]. Nevertheless, anthocyanin still exhibited strong antioxidant ability in a given limitation. Therefore, high levels of strong oxidant should be avoided in the food processing and application.

The reducing agent affected the absolute content of anthocyanin significantly but showed no distinct differences with the extension of time (Figure 5). The lower concentration range of reducing agent $\mathrm{Na}_{2} \mathrm{SO}_{3}(0.02$ $\mathrm{mM}$ and $0.1 \mathrm{mM}$ ) presented a synergistic effect on anthocyanin but residual rate of anthocyanin decreased 
dramatically with increasing concentration $(\geq 0.5 \mathrm{mM})$ (Figure 5). These results indicated that anthocyanin was sensitive to reducing agent, and advisable concentration of the reducing agent was less than $0.1 \mathrm{mM}$. The previous study reported that the anthocyanin of kernel and cob in purple maize possessed excellent antioxidant activity and that low concentration of $\mathrm{Na}_{2} \mathrm{SO}_{3}$ affected the stability of anthocyanin inapparently [12,17]. Here, we also confirmed these properties of anthocyanin in SDM kernels, but the synergistic mechanism was still unclear.

\subsection{Effects of Soluble Sugar and Food Additives Analysis}

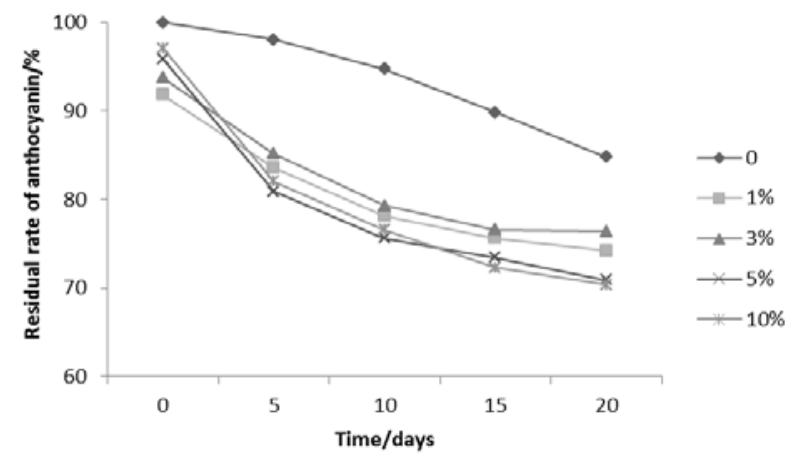

Figure 6. Change of residual rate of anthocyanin with time under different densities of glucose

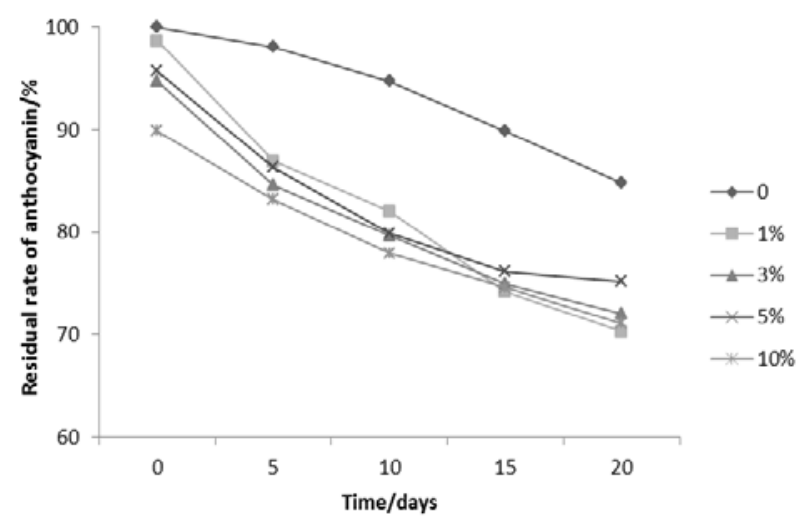

Figure 7. Change of residual rate of anthocyanin with time under different densities of sucrose

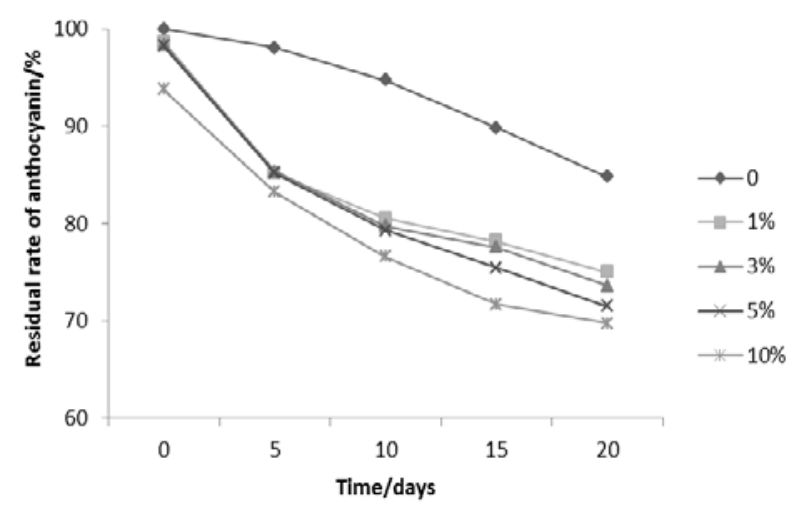

Figure 8. Change of residual rate of anthocyanin with time under different densities of maltose

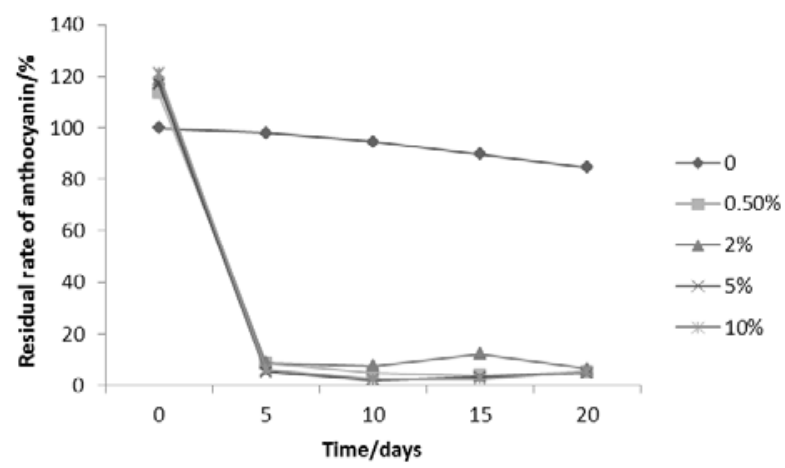

Figure 9. Change of residual rate of anthocyanin with time under different densities of ascorbic acid

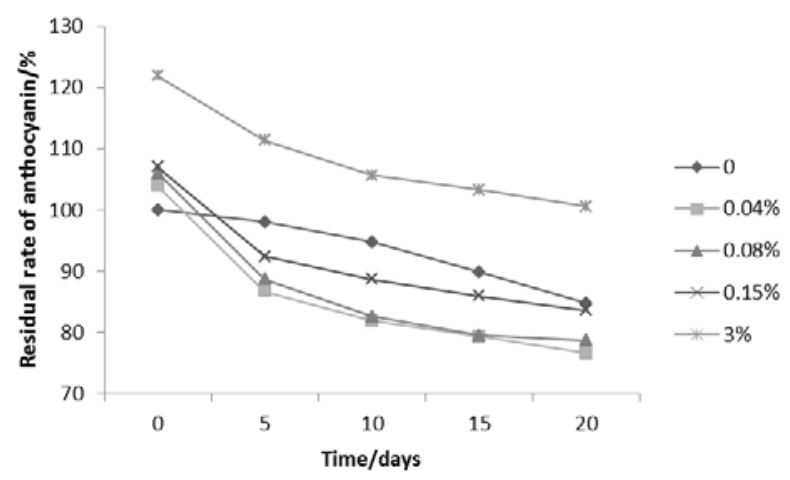

Figure 10. Change of residual rate of anthocyanin with time under different densities of citric acid

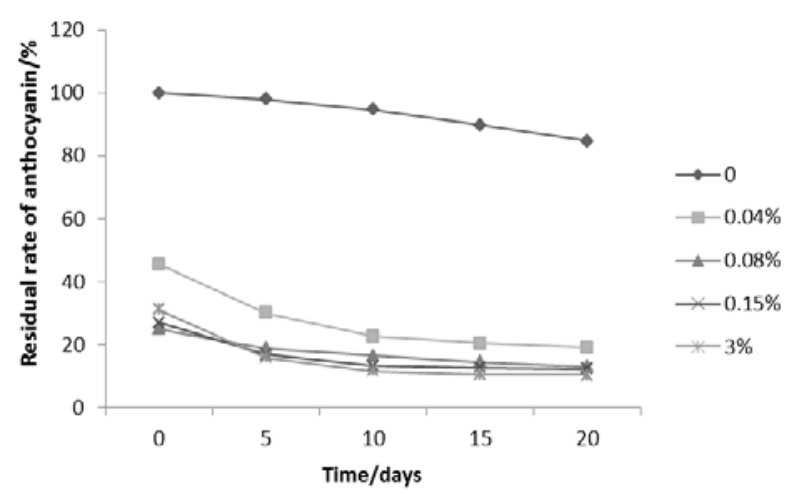

Figure 11. Change of residual rate of anthocyanin with time under different densities of benzoic acid

It had already been known that sugars had a positive effect on anthocyanin biosynthetic pathway in some plant [26]. Here, the effects of glucose, sucrose and maltose on anthocyanin were analysed (Figure 6, Figure 7, Figure 8). Compared with the control group, residual rates of anthocyanin for all three soluble sugars changed similarly with the time changing and the approximate $14 \%$ of residual rate of anthocyanin was decreased for all samples and all time points. These results suggested that the effects of soluble sugar on anthocyanin were inapparent and soluble sugar could be added in the practical application. Similarly, the effects of food additives on anthocyanin were exploited based on different concentrations of ascorbic acid, citric acid and benzoic acid (Figure 9, Figure 10, Figure 11). The ascorbic acid and citric acid exhibited a synergistic impact on anthocyanin in brief time, the mechanism was still unclear. With the extension of 
time, no obvious change of the residual rate was observed in the citric acid test. In contrast, the anthocyanin was seriously damaged by ascorbic acid and the residual rate decreased sharply because $\mathrm{H}_{2} \mathrm{O}_{2}$ would be produced after ascorbic acid and benzoic acid were oxidated (Figure 9, Figure 11). Moreover, although the residual rate of anthocyanin reduced heavily and immediately as benzoic acid was added, the absolute quantity of reducing showed no conspicuous differentiation (Figure 11). In a word, anthocyanin should be avoided contacting with ascorbic acid and benzoic acid but citric acid could be added appropriately.

\subsection{Effects of Metal Ions on Anthocyanin Absorbance}

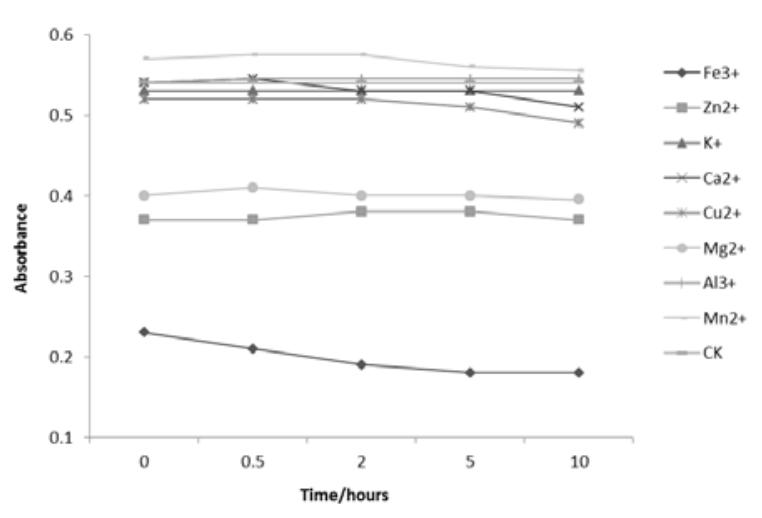

Figure 12. The effect of metal ions on absorbance of anthocyanin

The impacts of all the metal ions on anthocyanin were transient and had no remarkable changes with the extension of time (Figure 12). Ions of $\mathrm{Ca}^{2+}, \mathrm{Al}^{3+}, \mathrm{K}^{+}, \mathrm{Cu}^{2+}$ showed no obvious effect while $\mathrm{Mn}^{2+}$ exhibit positive effect on anthocyanin absorbance. The ions of $\mathrm{Mg}^{2+}, \mathrm{Zn}^{2+}$, $\mathrm{Fe}^{3+}$ made the absorbance decrease significantly and the largest degrees of decreasing was led by $\mathrm{Fe}^{3+}$. These results suggested that it was necessary for anthocyanin solution to avoid contacting with $\mathrm{Fe}, \mathrm{Zn}$, and $\mathrm{Mg}$ in saving. However, it could be stored in containers made of aluminum. As reported previously, metal ions of $\mathrm{Na}^{+}, \mathrm{Al}^{3+}$ weakly affected the stability of anthocyanin and low concentration of $\mathrm{K}^{+}$affected partly, but $\mathrm{Ca}^{2+}$ could make absorbance of anthocyanin reduce obviously [17]. Apart from this, it was confirmed that metal ions of $\mathrm{Zn}^{2+}, \mathrm{Mg}^{2+}$ also seriously affected anthocyanin in this research.

The metal ions exhibited different effects on anthocyanin based on the property of ortho-position hydroxyl structure of anthocyanin. In the anthocyanin solution, the compound with binary effects on the structure stability of anthocyanin was formed with metal ions [19]. In our study, a kind of destructive effect compound might be formed with metal ions of $\mathrm{Zn}^{2+}, \mathrm{Fe}^{3+}$, $\mathrm{Mg}^{2+}$ and weakened the structure stability of anthocyanin. On the other hand, a sort of compound with protective and synergistic effects on anthocyanin might be produced by interacting with metal ions of $\mathrm{Mn}^{2+}, \mathrm{Al}^{3+}$ and stabilized the anthocyanin structures.

\section{Conclusion}

In this study, the extraction and characterization of anthocyanin in SDM kernels were described and the antioxidant property and the stability under acid solvent, different temperatures and light were elucidated. In conclusion, the anthocyanin in SDM kernels maintained sensitivity to reducing agents and insensitivity to soluble sugar; citric acid exhibited positive effect on the stability of anthocyanin whereas benzoic acid and ascorbic acid negative; metal ions of $\mathrm{K}^{+}, \mathrm{Ca}^{2+}, \mathrm{Cu}^{2+}, \mathrm{Al}^{3+}, \mathrm{Mn}^{2+}$ showed no obvious effect on the stability of anthocyanin while ions of $\mathrm{Fe}^{3+}, \mathrm{Sn}^{2+}, \mathrm{Mg}^{2+}$ obvious. Moreover, the metal ions of $\mathrm{Zn}^{2+}$ seriously affected anthocyanin and couldn't coexist with anthocyanin. It is suggested to store anthocyanin in aluminium vessel.

\section{Acknowledgements}

This work was funded by the Chongqing key scientific and technological project (cstc2012ggC80003, cstc2012ggC80006, cstc2012ggC80007).

\section{Statement of Competing Interests}

The authors have no competing interests.

\section{References}

[1] Obi, Frederick Otunuya, Usenu, Ighofimoni Afonughe, \& Osayande, Julie Osaretin. (1998). Prevention of carbon tetrachloride-induced hepatotoxicity in the rat by $\mathrm{H}$. rosasinensis anthocyanin extract administered in ethanol. Toxicology, 131 (2), 93-98.

[2] Lee, David W, \& Gould, Kevin S. (2002). Anthocyanins in leaves and other vegetative organs: an introduction. Advances in Botanical Research, 37, 1-16.

[3] Nam, Seok Hyun, Choi, Sun Phil, Kang, Mi Young, Koh, Hee Jong, Kozukue, Nobuyuki, \& Friedman, Mendel. (2006). Antioxidative activities of bran extracts from twenty one pigmented rice cultivars. Food Chemistry, 94 (4), 613-620.

[4] Merzlyak, Mark N, \& Hendry, GAF. (1994). Free radical metabolism, pigment degradation and lipid peroxidation in leaves during senescence. PROCEEDINGS-ROYAL SOCIETY OF EDINBURGH B, 102, 459-459.

[5] Wang, Hong, Cao, Guohua, \& Prior, Ronald L. (1997). Oxygen radical absorbing capacity of anthocyanins. Journal of Agricultural and Food Chemistry, 45 (2), 304-309.

[6] Wrolstad, RE. (2004). Anthocyanin pigments-Bioactivity and coloring properties. Journal of Food Science, 69 (5), C 419-C 425.

[7] Wang, Li-Shu, \& Stoner, Gary D. (2008). Anthocyanins and their role in cancer prevention. Cancer letters, 269 (2), 281-290.

[8] Frankel, E. N. (1993). In Search of Better Methods to Evaluate Natural Antioxidants and Oxidative Stability in Food Lipids. Trends Food Sci. Technol. 4, 220-225.

[9] Guohua, Cao, Robert, M. Russell, Neal, Lischner and Ronald L. Prior. (1998). Serum Antioxidant Capacity Is Increased by Consumption of Strawberries, Spinach, Red Wine or Vitamin C in Elderly Women. J. Nutr. 128: 2383-2390.

[10] Hollman, PC, \& Katan, MB. (1999). Dietary flavonoids: intake, health effects and bioavailability. Food and chemical toxicology: an international journal published for the British Industrial Biological Research Association, 37 (9-10), 937.

[11] Shao-qian, CAO, Liang, LIU, \& Si-yi, PAN. (2011). Thermal degradation kinetics of anthocyanins and visual color of blood orange juice. Scientia Agricultura Sinica 10 (12).

[12] Yang, Zhendong, \& Zhai, Weiwei. (2010). Identification and antioxidant activity of anthocyanins extracted from the seed and cob of purple corn (Zea mays L.). Innovative Food Science \& Emerging Technologies, 11 (1), 169-176. 
[13] Ruenroengklin, Neungnapa, Yang, Bao, Lin, Hetong, Chen, Feng, \& Jiang, Yueming. (2009). Degradation of anthocyanin from litchi fruit pericarp by H2O2and hydroxyl radical. Food Chemistry, 116 (4), 995-998.

[14] JunNa, Zhang, \& LiXin, Huang. (2009). Effect of illumination and temperature on the stability of purple corn anthocyanin. Modern Food Science and Technology, 25 (11), 1282-1285, 1257.

[15] Zhao, Xiaoyan, Corrales, Margarita, Zhang, Chao, Hu, Xiaosong, Ma, Yue, \& Tauscher, Bernhard. (2008). Composition and thermal stability of anthocyanins from Chinese purple corn (Zea mays L.). Journal of agricultural and food chemistry, 56 (22), 10761-10766.

[16] Yang, Zhendong, Han, Yonbin, Gu, Zhenxin, Fan, Gongjian, \& Chen, Zhigang. (2008). Thermal degradation kinetics of aqueous anthocyanins and visual color of purple corn (Zea mays L.) cob. Innovative Food Science \& Emerging Technologies, 9 (3), 341347.

[17] XIAO, Xiang, \& WANG, Li-ya. (2012). Study of the Stability of Purple Corn Pigment. Modern Food Science and Technology, 1, 006.

[18] Xu, JR, Zhang, MW, Liu, XH, Liu, ZX, Zhang, RF, Sun, Ling, \& Qiu, LJ. (2006). Correlation between antioxidation, and content of total phenolics and anthocyanin in black soybean accessions. Scientia Agricultura Sinica, 39 (8), 1545-1552.

[19] JianXia, Sun, Yan, Zhang, Xiaosong, HU, JiHong, Wu, \& XiaoJun, Liao. (2009). Structural stability and degradation mechanisms of anthocyanins. Scientia Agricultura Sinica, 42 (3), 996-1008.
[20] QIN Hong-Ni, YAN Meng, WANG Zhao-Hui, GUO Ying, WANG Hui, SUN Hai-Yan, LIU Zhi-Zhai, and, \& Yi-Lin, CAI. (2012). QTL Mapping for Anthocyanin and Melanin Contents in Maize Kernel. ACTA AGRONOMICA SINICA, 38 (02), 275-284.

[21] Maccarone, Emanuele, MACCARRONE, ANGELO, \& RAPISARDA, PAOLO. (1985). Stabilization of anthocyanins of blood orange fruit juice. Journal of Food Science, 50 (4), 901-904.

[22] Özkan, Mehmet. (2002). Degradation of anthocyanins in sour cherry and pomegranate juices by hydrogen peroxide in the presence of added ascorbic acid. Food Chemistry, 78 (4), 499-504.

[23] De Pascual-Teresa, Sonia, \& Sanchez-Ballesta, Maria Teresa. (2008). Anthocyanins: from plant to health. Phytochemistry reviews, 7 (2), 281-299.

[24] Tonon, Renata V, Brabet, Catherine, \& Hubinger, Míriam D. (2010). Anthocyanin stability and antioxidant activity of spraydried açai (Euterpe oleracea Mart.) juice produced with different carrier agents. Food Research International, 43 (3), 907-914.

[25] ISAAC, RABINO, \& ALBERTO L., MANCINELLI. (1986). Light, Temperature, and Anthocyanin Production. Plant Physiol. 81, 922-924.

[26] Solfanelli, Cinzia, Poggi, Alessandra, Loreti, Elena, Alpi, Amedeo, \& Perata, Pierdomenico. (2006). Sucrose-specific induction of the anthocyanin biosynthetic pathway in Arabidopsis. Plant Physiology, 140 (2), 637-646. 